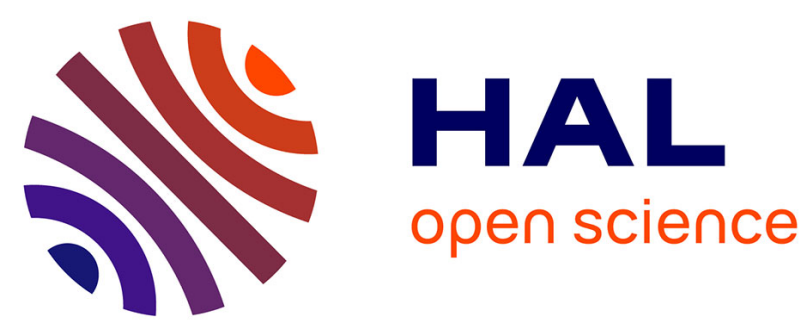

\title{
Viscous Effects on Inertial Drop Formation
}

\author{
Antoine Deblais, M. A. Herrada, I. Hauner, K. P. Velikov, T. van Roon, \\ Hamid Kellay, Jens Eggers, D. Bonn
}

\section{To cite this version:}

Antoine Deblais, M. A. Herrada, I. Hauner, K. P. Velikov, T. van Roon, et al.. Viscous Effects on Inertial Drop Formation. Physical Review Letters, 2018, 121 (25), pp.254501. 10.1103/physrevlett.121.254501 . hal-01989855

\section{HAL Id: hal-01989855 https://hal.science/hal-01989855}

Submitted on 22 Jan 2019

HAL is a multi-disciplinary open access archive for the deposit and dissemination of scientific research documents, whether they are published or not. The documents may come from teaching and research institutions in France or abroad, or from public or private research centers.
L'archive ouverte pluridisciplinaire HAL, est destinée au dépôt et à la diffusion de documents scientifiques de niveau recherche, publiés ou non, émanant des établissements d'enseignement et de recherche français ou étrangers, des laboratoires publics ou privés. 


\title{
Viscous Effects on Inertial Drop Formation
}

\author{
A. Deblais, ${ }^{1}$ M. A. Herrada, ${ }^{2}$ I. Hauner, ${ }^{1}$ K. P. Velikov, ${ }^{1,3}$ T. van Roon, ${ }^{4}$ H. Kellay, ${ }^{5}$ J. Eggers, ${ }^{6}$ and D. Bonn ${ }^{1}$ \\ ${ }^{1}$ Van der Waals-Zeeman Institute, Institute of Physics, University of Amsterdam, 1098XH Amsterdam, Netherlands \\ ${ }^{2}$ Depto. de Mecánica de Fluidos e Ingeniería Aeroespacial, Universidad de Sevilla, E-41092 Sevilla, Spain \\ ${ }^{3}$ Unilever R\&D Vlaardingen, Olivier van Noortlaan 120, 3133 AT Vlaardingen, Netherlands \\ ${ }^{4}$ Technology Centre, University of Amsterdam, 1098XH Amsterdam, Netherlands \\ ${ }^{5}$ Laboratoire Ondes et Matiere d'Aquitaine, UMR 5798 CNRS-U. Bx, Universite de Bordeaux, \\ 351 cours de la Liberation 33405, Talence, France \\ ${ }^{6}$ School of Mathematics, University of Bristol, University Walk, Bristol BS8 1 TW, United Kingdom
}

(Received 6 June 2018; revised manuscript received 10 September 2018; published 21 December 2018)

\begin{abstract}
The breakup of low-viscosity droplets like water is a ubiquitous and rich phenomenon. Theory predicts that in the inviscid limit one observes a finite-time singularity, giving rise to a universal power law, with a prefactor that is universal for a given density and surface tension. This universality has been proposed as a powerful tool to determine the dynamic surface tension at short time scales. We combine high-resolution experiments and simulations to show that this universality is unobservable in practice: in contrast to previous studies, we show that fluid and system parameters do play a role; notably a small amount of viscosity is sufficient to alter the breakup dynamics significantly.
\end{abstract}

DOI: 10.1103/PhysRevLett.121.254501

Drop formation is a common phenomenon that occurs in a broad range of industrial processes such as inkjet printing, spraying, and cooling. In a typical laboratory experiment, a drop is formed at the end of a syringe tip, and its detachment is filmed using a high-speed camera. Surface tension drives the flow that shrinks the diameter of the filament connecting the drop to the syringe, and both fluid inertia and fluid viscosity slows it down. The filament can be observed to thin in a nonuniform way, which eventually leads to the pinch-off of the drop (see Fig. 1) occurring at a critical time $t_{c}$. In the case of negligible viscosity, based on the idea that the minimum neck radius $R_{\min }$ is the only characteristic length scale close to pinch-off, one predicts the power law $[1,2]$

$$
R_{\min }=A\left(\frac{\gamma}{\rho}\right)^{1 / 3}\left(t_{c}-t\right)^{2 / 3}
$$

where $\gamma$ and $\rho$ are the surface tension and the density of the liquid, respectively.

Potential flow simulations and similarity theory [3-5] confirm Eq. (1), with $A=0.717 \ldots$ a universal constant [4-6]. The profile near the pinch point converges toward a double cone, one set inside the other with opening angles of $18^{\circ}$ and $112^{\circ}[4,7]$ [cf. Fig. 3(c) below]. This means that eventually the profile must overturn [3,4,8], as indeed seen in the last stages of pinch-off, shown in the last two panels of the simulations shown in Fig. 1. It is expected that the above potential flow theory applies as long as the viscous length scale $\ell_{\nu}=\nu^{2} \rho / \gamma$ is much smaller than $R_{\min }[6,9]$, where $\nu$ is the kinematic viscosity of the liquid. These trends have been confirmed by experiment and simulation [7,10,11], and Eq. (1) has been proposed as the basis of a method to measure surface tension on a millisecond timescale $[12,13]$.

However, a careful analysis of past measurements in the inertial regime reveals a considerable variation of the prefactor $A$. Some of the values found in the literature are $0.45-0.5$ [10], 0.55 [14], 0.57-0.68 [7], or 0.97 [15].

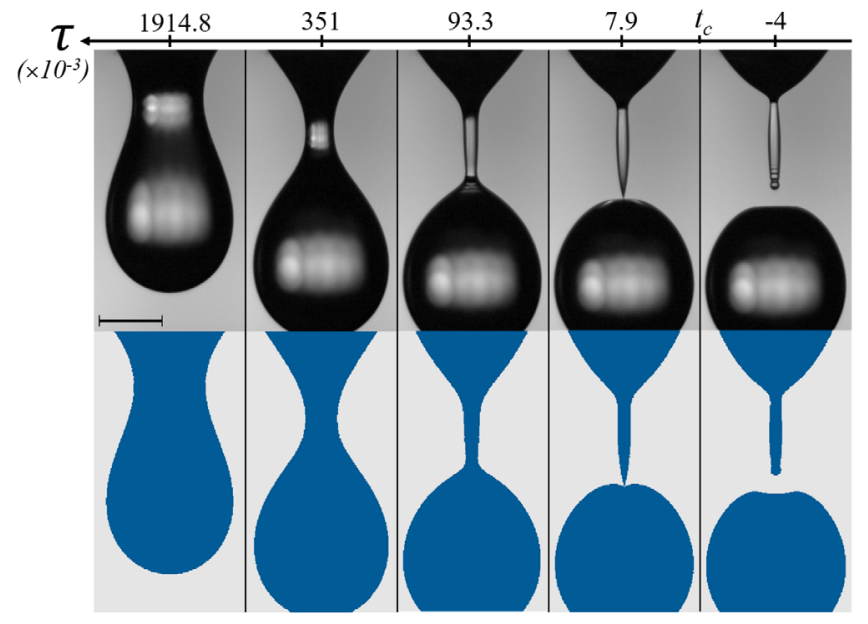

FIG. 1. High-speed image sequence (upper panel) of a drop of water dripping from a faucet of $2 \mathrm{~mm}$ in radius. Lower panel: numerical simulation of the same problem using the Basilisk code, showing $2 \mathrm{D}$ cuts through the axis. Note the overturning of the upper surface of the main drop in the last two frames. The scale bar represents $R_{0}=2 \mathrm{~mm}$. The dimensionless time to pinch-off $\tau=\left(t_{c}-t\right) / t^{*}$ is indicated on the top of the figure. 
Recently there has been a growing appreciation of the fact that the approach to asymptotic power laws like Eq. (1) can be slow [16-18], and may pass through one or several transient regimes before the final universal regime is reached.

One obstacle to resolving the inconsistency in the measured values of $A$ is that at the very last instant before the breakup, the drop profile overturns [7,19]. As seen in the cross sections produced from numerical simulation (lower row of Fig. 1, 4th panel), the neck ends in a sharp tip, which lies inside an indentation formed on the top of the drop. The corresponding experimental image, taken from the side, shows a projection onto the plane, which produces a flat top of the drop, where overturning has occurred; the end of the neck is obscured. This means that the neck radius can only be measured before overturning, at a typical size $R_{\text {min }} \simeq 5 \mu \mathrm{m} \mathrm{[20].}$

Since the asymptotic value of $A$ refers to the profile having attained its final double cone shape after overturning occurs, conventional optical techniques are incapable of determining its asymptotic value. To circumvent these optical limitations, and to probe length scales well below the optical resolution, Burton and Taborek [11] measured the electrical resistance $r$ through the drop, whose main contribution comes from where the neck is thinnest, and thus scales like the resistivity $\rho_{r}$ of the material, divided by $R_{\min }$. The resistance was found to scale in accordance with Eq. (1) down to nanometer length and nanosecond time scales. However, in order to calculate $R_{\text {min }}$ from $r$, the shape of the interface is needed. An approximate formula leads to a value of $A=0.10$ [11], much smaller than the theoretical expectation or the result of previous optical measurements.

In this Letter, to arrive at a definitive answer as to the validity of the scaling law (1), we use a combination of experiment (using both optical and electrical measurements) and high resolution numerical simulations. We show that the correct asymptotic value of $A$ is unobservable for all practical purposes. On the one hand, the initial dynamics leaves its imprint and is slow to converge onto Eq. (1). On the other hand, the effect of viscosity is felt even if $R_{\min }$ is greater than $\ell_{\nu}$ by several orders of magnitude, as is usually the case, e.g., for water, with $\ell_{\nu}=14 \mathrm{~nm}$.

Different liquids allow us to perform pinch-off experiments with different ratios $\gamma / \rho$. We used decane from Sigma Aldrich and highly ultrapure water from a Millipore Milli-Q system. Surface tensions $\gamma$ were verified independently using the pendant drop method (Kruss Easy Drop); we used $\gamma_{\text {water }}=72 \mathrm{mN} \mathrm{m}^{-1}$ and $\gamma_{\text {decane }}=24 \mathrm{mN} \mathrm{m}^{-1}$. A syringe pump (Harvard Apparatus) supplied the solutions at the needle tip at a slow rate (needle tip radii: $R_{0}=0.2$ and $2 \mathrm{~mm}$ ), so we can observe the detachment of a single drop at a time.

A high-speed camera (Phantom V701, Vision Research) equipped with a microscope tube lens (Microscope objectives Zeiss, $4 \times$ to $10 \times$ magnification and Navitar $12 \times$ ) recorded pinch-off events at up to 100000 frames per second with a spatial resolution as low as $1.6 \mu \mathrm{m} /$ pixel. We paid particular attention to a stable setup, and used microscope objectives with very high contrast and low depth of field. Drop profiles were extracted using a homemade MATLAB routine, from which $R_{\min }(t)$ was found.

The numerical technique used to simulate the pinch-off of low-viscosity pendant drops is a variation of that described in detail in Ref. [21]. It was previously applied to study the effect of surface contamination on the dynamics of pendant drops [22]. The main features of the method are the use of an analytical mapping to convert the numerical domain into a rectangular one and implementation of the Newton procedure to solve the nonlinear system of equations needed for a fully implicit treatment of the discretized equations. The numerical Jacobian required by the method is built with the help of analytical functions and the collocation matrix provided by the spatial discretization of the mapped domain. With this method we can accurately capture the dynamics of the pendant drops until the appearance of overturning.

However, the analytical mapping fails after overturning and, like the experimental optical measurements, cannot be used to study the subsequent dynamics. Therefore, closer to pinch-off and in order to compare with the electrical measurements in the mercury liquid bridge experiment, we used a numerical scheme based on a finite element volume-of-fluid (VOF) method provided by the "Basilisk" software [23]. It uses QuADTREES [24] to allow efficient adaptive grid refinement of the interface close to the pinchoff region (see Supplemental Material, Sec. A [25]).

The main results of our fluid pinch-off experiments are presented in Fig. 2 for decane and water, using two different capillary sizes to assess the influence of the initial conditions. In the limit of slow dripping, the dimensionless parameters of the experiment are the Bond number $\mathrm{Bo}=\rho g R_{0}^{2} / \gamma$ and the Ohnesorge number $\mathrm{Oh}=\sqrt{\ell_{\nu} / R_{0}}=\nu / \sqrt{\rho R_{0} \gamma}$. We calculated instantaneous values of $A$ from the slope of $\left(R_{\min } / R_{0}\right)^{3 / 2}=A^{3 / 2} \tau$ as a function of the dimensionless breakup time $\tau=\left(t_{c}-t\right) / t^{*}$, with $t^{*}=\sqrt{\rho R_{0}^{3} / \gamma}$ the capillary time. This procedure does not involve a determination of the extrapolated time to pinch-off $t_{c}$ for the determination of the prefactor $A$, and therefore does not make an assumption on the validity of Eq. (1).

Surprisingly, for both liquids, over the range $2 \times 10^{-3}<$ $\tau<1$ before overturning (indicated by a cross), the prefactor varies nonmonotonically between 0.4 and 0.6 . In addition, our mapping simulations perfectly capture the experimental variation of the prefactor in all the cases presented here until overturning. To follow the dynamics of thinning beyond the point of overturning, we used Basilisk simulations, which agree very well with data before overturning. Although $R_{\min }$ is still greater than $\ell_{\nu}$ by more than 

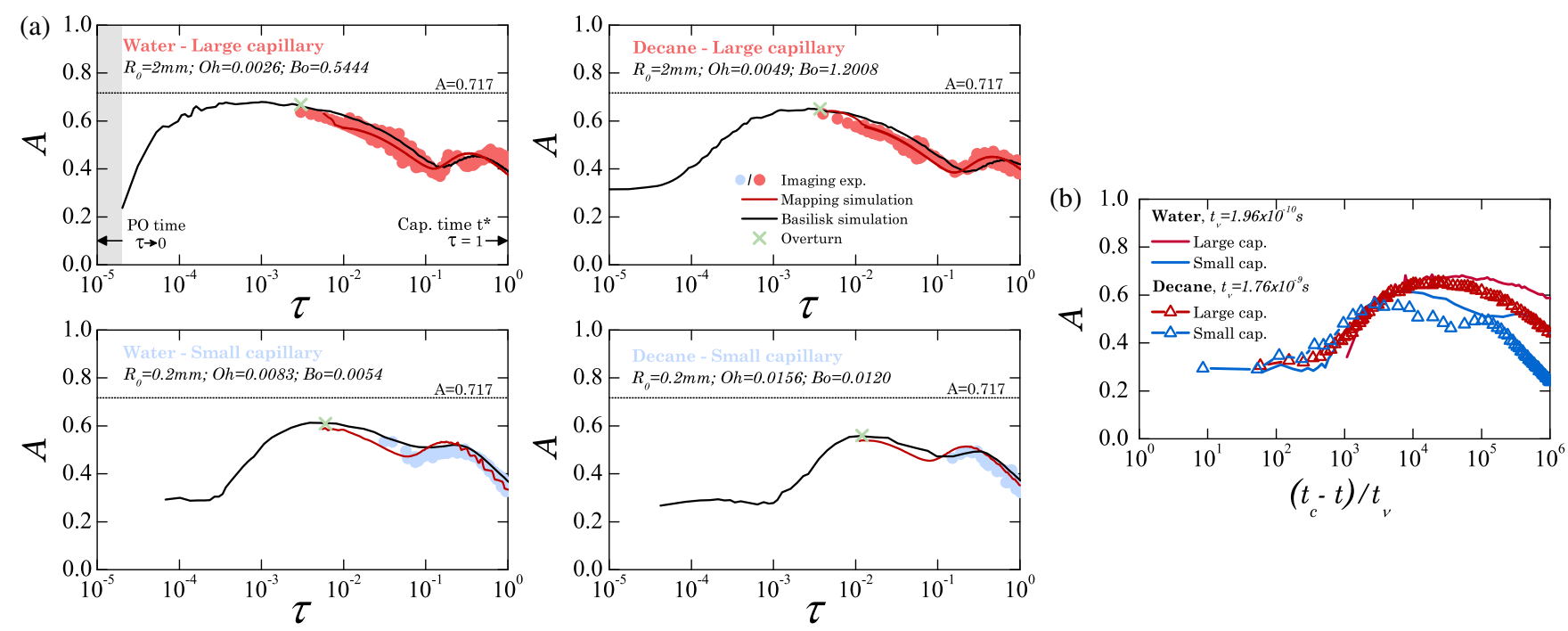

FIG. 2. (a) Prefactor $A$ as a function of the dimensionless time to pinch-off $\tau$ for water and decane for different values of the capillary size $R_{0}$, the Ohnesorge number Oh, and Bond number Bo. Symbols indicate experimental results whereas solid lines were obtained from two different simulation techniques: mapping (red) and Basilisk (black). After a nonmonotonic variation, $A$ reaches a maximum value and starts to decrease on account of viscous effects. The theoretical prediction for $A$ is depicted as a horizontal dashed line. (b) The prefactor $A$ is plotted as a function of the characteristic viscous dimensionless timescale $\left(t_{c}-t\right) / t_{\nu}$ : all the curves (from basilisk simulation) collapse into a single master curve for late times, showing that the drop of the prefactor results from viscous effects.

2 orders of magnitude, $A$ starts to decrease on account of viscous effects (as an example, see the case of Decane for large capillary at $\tau \sim 10^{-3}$ in Fig. 2). To confirm that the drop of the prefactor as a result of viscous effects is physical, we plot in Fig. 2(b) the prefactor $A$ as a function of the dimensionless time $\left(t_{c}-t\right) / t_{\nu}$, where $t_{\nu}=\nu^{3} \rho^{2} / \gamma^{2}$ is the characteristic viscous timescale. We find that this collapses the drop of the prefactor $A$ for late times, confirming the physical origin of this behavior. Thus even for the smallest Ohnesorge numbers the prefactor never reaches the asymptotic value of 0.70 reported in Ref. [10]. This observation is well confirmed in the Supplemental Material [25], where we simulate two artificial liquids with the same control parameters except for $\mathrm{Oh}$, which is varied by almost 1 order of magnitude (see Supplemental Material [25], Sup. Fig. 3).

To see whether the asymptotic state can be seen experimentally, we push our experimental investigation closer to pinch-off by measuring the resistance of a mercury bridge [see Figs. 3(a),3(b)]. We use mercury for its low resistivity $\rho_{r}$ and on account of its small viscous length scale $\ell_{\nu}=4.2 \times 10^{-10} \mathrm{~m}$, even smaller than that of water. Our electrical circuit consists of a voltage-biased voltage divider [11]. A mercury capillary bridge is formed between two copper electrodes (radius $R_{0}=1 \mathrm{~mm}$ ); the upper one can be translated vertically with a step motor. A droplet of volume $V_{0}=5 \mu \mathrm{L}$ of fresh mercury is deposited on the lower electrode. Prior to measurements, the mercury surface is cleaned with concentrated sulfuric acid $\left(\mathrm{H}_{2} \mathrm{SO}_{4}\right)$.

To prevent any further oxidation of the superficial layer of mercury with air during the capillary bridge breakup, the entire setup is placed in a chamber flushed continuously with pure nitrogen. In fact, we believe surface contamination to be of minor importance for the asymptotic regime, as the new surface is created faster than impurities can arrive [28]. The maximum bandwidth of our oscilloscope (RTO 1024, Rohde and Schwartz) is limited to $2 \mathrm{GHz}$, with a sampling rate of $10 \times 10^{9}$ samples/s. The high-frequency response of our system is evaluated by bringing the two copper electrodes together in the absence of mercury, which gives a step function with a rise time of 300 ps. The recorded oscilloscope data represent the output voltage $V_{s}(\tau)$ of the voltage divider circuit [11], which decreases as the fluid neck becomes thinner [cf. Fig. 3(b)]. The resistance $r_{\mathrm{Hg}}$ of the mercury bridge is found from $r_{\mathrm{Hg}}=r_{\text {scope }}\left(V_{\text {in }} / V_{s}-1\right)$, with $r_{\text {scope }}=50 \Omega$ the internal resistance of the scope and $V_{\text {in }}=1 \mathrm{~V}$ the tension supplied by the voltage generator (see Supplemental Material [25]).

To compare the measured $r$ to numerical simulation, we numerically calculate the current flow through the computed profile, which is shown in Fig. 3(c) for different values of $R_{\min }$ and compared to the profile of the inviscid similarity solution [5]. At $R_{\min }=10^{-4}$ the computed profile comes very close to the similarity profile, but then overshoots to form an even larger angle than predicted on the drop side. To compute the resulting electrical resistance, we solve Laplace's equation inside the liquid, with Neumann boundary conditions on the surface [29] (see Supplemental Material, Sec. B [25] for more details). The resulting dimensionless resistance is shown in Fig. 3(d), and indeed continues to evolve down to the smallest scales considered. Owing to the continuous evolution of the 


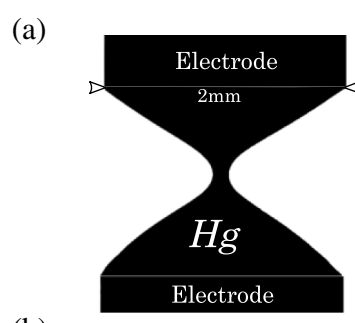

(b)

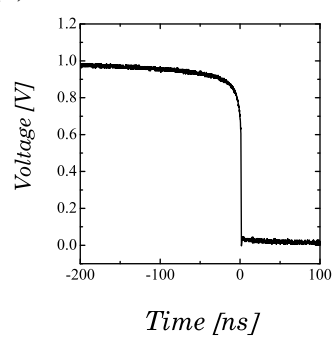

(c)

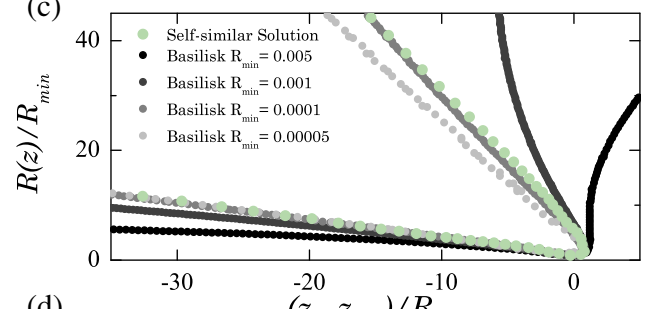

(d)

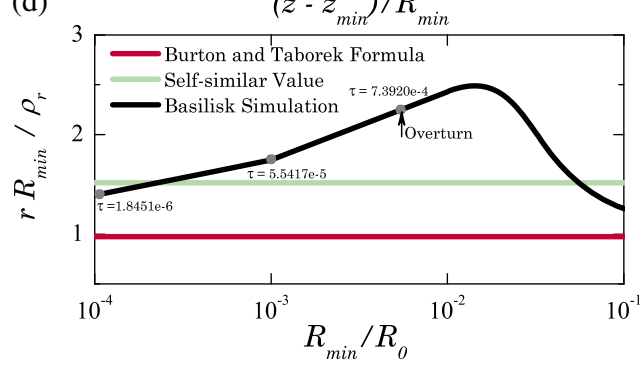

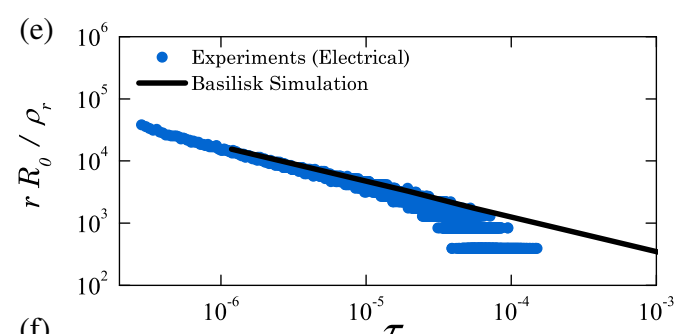

(f)

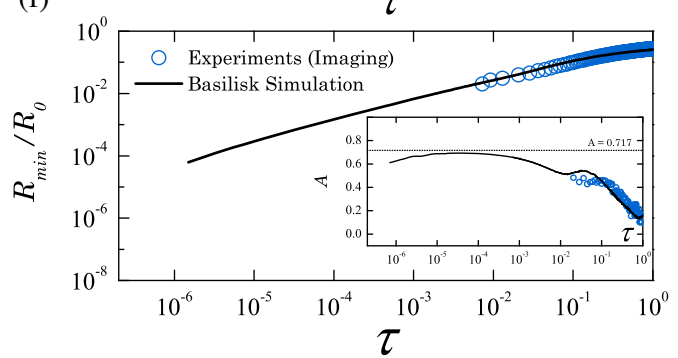

FIG. 3. Breakup dynamics of a mercury bridge $\left(\mathrm{Oh}=6.0437 \times 10^{-4}\right)$ from the millimeter to the nanometer scale. (a) Electrical resistance of a mercury capillary bridge between two electrodes as function of time. The upper electrode moves upwards, initiating breakup; the bridge's spatial profile is recorded simultaneously using high-speed imaging. (b) Typical measurement of voltage $V_{s}$ over the divider circuit as a function of time during the breakup process (note the nanometer timescale); at breakup (denoted here as $t=0$ ) the voltage decreases suddenly. This is used to trigger fast imaging. (c) The shape of the liquid bridge $R(z)$ is computed from a Basilisk simulation, allowing us to calculate the resistance $r$ of the filament in time. (d) The resistance as function of minimum radius, compared to the asymptotic value of the inviscid solution, and the estimate by Burton and Taborek. (e) The measured resistance of the liquid bridge data is well described by the simulations very close to the pinch-off point. (f) The dynamics of thinning of the liquid bridge before overturning recorded by imaging techniques is also well captured by Basilisk simulations. In inset, the prefactor $A$ of mercury is plotted as a function of the dimensionless time to pinch-off $\tau$.

self-similar profile, the resistance does not converge to the value $r=1.51 \rho_{r} / R_{\min }$ found for the inviscid similarity solution, and is considerably higher than $r=0.97 \rho_{r} / R_{\min }$ estimated in Ref. [11]. We therefore compare directly the simulated resistivity with that obtained by the experiment.

The electrical setup is coupled to the fast camera, triggered by the oscilloscope of the breakup event; this allows us to compare electrical with optical measurements in the same experiment. The optical measurement works over the range $0.05 \mathrm{~ms}<t<0.1 \mathrm{~s}$ and $10 \mu \mathrm{m}<$ $R_{\text {min }}<1 \mathrm{~mm}$, while the electrical measurements have much higher resolution: $1 \mathrm{~ns}<t<0.7 \mu$ s and $10 \mathrm{~nm}<$ $R_{\min }<10 \mu \mathrm{m}$. Our electrical resistance measurements are presented in Fig. 3(e) and show quantitative agreement with the simulation on timescales well below any where such a comparison has been made so far. Only farther away from pinch-off does our resistance measurement deviate from simulation, as other electrical resistance effects start come into play when the resistance is no longer dominated by the neck region alone. However, for longer times we can use the optical measurement done in the same experiment; these also agree perfectly with the simulations [cf. Fig. 3(f)]. The electrical experiments alone do not allow us to unambiguously show the nonuniversality since the neck shape is not known a priori. The simulations do provide the neck shape and by comparing to the experiment these allow us to unambiguously determine the radius at each time and therefore the prefactor $A$. The agreement with the experiments provides the necessary evidence that the numerics work well. Thus, taken together, the measurements and simulations give the results for the prefactor $A$ over a large range of length scales and timescales [see inset of Fig. 3(f)]. The comparison also shows that for low viscosity fluids such as mercury $\left(\mathrm{Oh}=6.0437 \times 10^{-4}\right)$, the prefactor remains close to the purely inviscid prediction until the effects of viscosity come into play.

In conclusion, we study drop pinch-off in cases where the inner (viscous) length scale is 5 (water) or 7 (mercury) orders of magnitude greater than the outer length scale $R_{0}$, both experimentally and computationally. We find that the dimensionless prefactor $A$ exhibits a complex, nonmonotonic behavior over many orders of magnitude in time, measured relative to the breakup time. We never found the prefactor to fully reach the asymptotic value of $A=0.717$. This indicates the existence of very slow transients, similar to transients reported for more viscous fluids $[17,18]$. However, in those experiments the theoretical value of the prefactor is reached eventually both for the viscous and viscous-inertial regimes [18], indicating that in our experiments the inertial transients are even slower and the asymptotic value is not even reached for nanometric length and nanosecond time scales; for smaller length scales and timescales one may even wonder whether a continuum hydrodynamics description remains valid. Apart from the fundamental interest, the full understanding of the slow time dependence of $A$ is crucial to measure the dynamic 
surface tension of liquids: if one wishes to determine this, one has to take into account that fluid and system parameters play an important role.

We are grateful to David Leppinen for sharing numerical data of similarity profiles from Ref. [5]. J. E. acknowledges support from the Leverhulme Trust through International Academic Fellowship IAF-2017-010. H. K. thanks the Institut Universitaire de France for partial support. M. A. H. thanks the Ministerio de Economía y competitividad for partial support under the Project No. DPI201678887-C3-1-R. This project has been partially supported in part by Unilever R\&D Vlaardingen.

A. D. and M. A. H. contributed equally to this work.

[1] D. H. Peregrine, G. Shoker, and A. Symon, J. Fluid Mech. 212, 25 (1990).

[2] J. Eggers, Phys. Rev. Lett. 71, 3458 (1993).

[3] Y.-J. Chen and P. H. Steen, J. Fluid Mech. 341, 245 (1997).

[4] R. F. Day, E. J. Hinch, and J. R. Lister, Phys. Rev. Lett. 80, 704 (1998).

[5] D. Leppinen and J. R. Lister, Phys. Fluids 15, 568 (2003).

[6] J. Eggers and E. Villermaux, Rep. Prog. Phys. 71, 036601 (2008).

[7] J. R. Castrejon-Pita, A. A. Castrejon-Pita, E. J. Hinch, J. R. Lister, and I. M. Hutchings,Phys. Rev. E 86, 015301(R) (2012).

[8] P. Lenard, Ann. Phys. (Berlin) 266, 209 (1887).

[9] J. R. Lister and H. A. Stone, Phys. Fluids 10, 2758 (1998).

[10] A. U. Chen, P. K. Notz, and O. A. Basaran, Phys. Rev. Lett. 88, 174501 (2002).

[11] J. C. Burton, J. E. Rutledge, and P. Taborek, Phys. Rev. Lett. 92, 244505 (2004).

[12] M. R. De Saint Vincent, J. Petit, M. Aytouna, J. P. Delville, D. Bonn, and H. Kellay,J. Fluid Mech. 692, 499 (2012).
[13] I. M. Hauner, A. Deblais, J. K. Beattie, H. Kellay, and D. Bonn, J. Phys. Chem. Lett. 8, 1599 (2017).

[14] M. P. Brenner, J. Eggers, K. Joseph, S. R. Nagel, and X. D. Shi, Phys. Fluids 9, 1573 (1997).

[15] J. C. Burton, J. E. Rutledge, and P. Taborek, Phys. Rev. E 75, 036311 (2007).

[16] J. Eggers, M. A. Fontelos, D. Leppinen, and J. H. Snoeijer, Phys. Rev. Lett. 98, 094502 (2007).

[17] J. R. Castrejón-Pita, A. A. Castrejón-Pita, S. S. Thete, K. Sambath, I. M. Hutchings, J. Hinch, J. R. Lister, and O. A. Basaran, Proc. Natl. Acad. Sci. U.S.A. 112, 4582 (2015).

[18] Y. Li and J. E. Sprittles, J. Fluid Mech. 797, 29 (2016).

[19] J. C. Burton, J. E. Rutledge, and P. Taborek, Phys. Rev. Lett. 92, 244505 (2004).

[20] J. C. Burton and P. Taborek, Phys. Fluids 19, 102109 (2007).

[21] M. A. Herrada and J. M. Montanero, J. Comput. Phys. 306, 137 (2016).

[22] A. Ponce-Torres, J. M. Montanero, M. A. Herrada, E. J. Vega, and J. M. Vega, Phys. Rev. Lett. 118, 024501 (2017).

[23] http://basilisk.fr/.

[24] S. Popinet, Ocean Dyn. 61, 1261 (2011).

[25] See Supplemental Material at http://link.aps.org/ supplemental/10.1103/PhysRevLett.121.254501 for details on the validation of the numerical codes, numerical comput ance in the liquid bridge, and more details on the electrical setup, which includes Refs. [19,26,27].

[26] C. Ferrera, M. A. Herrada, J. M. Montanero, M. Torregrosa, and V. Shevtsova, Phys. Fluids 26, 012108 (2014).

[27] M. A. Herrada, J. M. López-Herrera, E. J. Vega, and J. M. Montanero, Phys. Fluids 23, 012101 (2011).

[28] M. Roché, M. Aytouna, D. Bonn, and H. Kellay, Phys. Rev. Lett. 103, 264501 (2009).

[29] W. R. Smythe, in Static and Dynamic Electricity (McGrawHill, New York, 1950). 